\section{OS RESÍDUOS DE SERVIÇOS DE SAÚDE - RSS E A QUESTÃO AMBIENTAL}

Adriana A. Mendes* Janaína F. F. Cintrão**

\section{Introdução}

O homem produz resíduos que podemos encontrar nas formas sólida, líquida e gasosa. Esta última forma de resíduo é responsável pela poluição do ar e decorre de vários fatores, sendo um dos principais, a industrialização, que é responsável, em grande parte, pela emissão de gases tóxicos, os quais comprometem tanto a qualidade do ar que respiramos, quanto a proteção da superficie da Terra e de todos os seus elementos abióticos, através de alterações na camada de ozônio.

Dentre os resíduos líquidos gerados pelo homem, os esgotos domiciliares representam o maior fator de poluição das águas, se não forem submetidos a processo de tratamento antes de serem despejados nos cursos de água

Já os resíduos sólidos, podem provocar alterações intensas não só no solo, como na água e no ar; se inadequadamente dispostos, além da possibilidade de causarem danos a todas as formas de vida, trazendo problemas que podem aparecer, com freqüência, anos depois da disposição inicial. Além disso, podem favorecer o aumento de vetores, que são veiculados ou reservatórios de certos patogênicos, o que pode aumentar o risco existente entre os resíduos sólidos para a saúde humana.

Nesse sentido, durante muito tempo, os resíduos de serviço de saúde RSS, foram denominados de "lixos hospitalares". A palavra "lixo" nos remete a uma situação de "sujeira", de produto de atividades domésticas ou industriais

* Mestranda do Programa de Pós-graduação - Mestrado em Desenvolvimento Regional e Meio Ambiente - Centro Universitário de Araraquara - UNIARA

** Docente do Programa de Pós-graduação - Mestrado em Desenvolvimento Regional e Meio Ambiente - Centro Universitário de Araraquara - UNIARA sem valor para a sociedade. Aurélio (1999), define a palavra lixo como "aquilo que se varre da casa, do jardim, da rua, e se joga fora, entulho". Comenta ainda que lixo é sinônimo de sujidade, sujeira, atribuindo a ele também como sendo coisas inúteis, velhas, sem valor que resultam de atividades domésticas, industriais e comerciais; mencionou também a existência do lixo atômico, lixo especial e lixo radioativo. Há também a questão do aspecto estético de um sistema inadequado de destinação dos resíduos, que é atualmente, muitas vezes inaceitável para a sociedade.

Sabemos que no passado, a matéria orgânica era o conteúdo exclusivo do lixo. Considerando que o índice populacional era baixo, o destino dos resíduos produzidos pelo homem era de fácil resolução, sendo enterrados, promovendo assim o controle dos vetores e a fertilização do solo.

Estudos demonstram que, com o passar dos tempos, o aumento populacional proporcionou mudanças na composição do lixo, assim, como as evoluções no campo produtivo e o aumento significativo do consumo dos produtos industrializados com suas embalagens de papel, papelão, plásticos e metais, foram surgindo novas definições de lixo.

Lima (2001), relata que a palavra "lixo" deixou de ser utilizada na década de 60 , onde recebeu dos sanitaristas a denominação de resíduos sólidos, que nada mais é do que o resultado de qualquer atividade natural quer seja ela de origem humana ou animal.

Mas uma preocupação continua a acompanhar todo o desenvolvimento humano: a redução do volume de resíduos e propostas de seu aproveitamento. Reduzir a geração de resíduos significa intervir com mudanças no comportamento social, entre elas, mudanças nos padrões de consumo assim como implantação de programas de coleta seletiva.

\section{Caracterização dos RSS}

A discussão dos resíduos sólidos é muito complexa, conferindo assim espaço para muitos trabalhos; porém, neste estudo será tratado especificamente sobre os resíduos de serviços de saúde - RSS, também chamados de lixo séptico, lixo hospitalar, a partir de uma revisão bibliográfica.

Segundo a Associação Brasileira de Normas Técnicas - ABNT (1989), a Norma Brasileira Registrada - NBR 10.004, os resíduos sólidos são definidos como: "Resíduos nos estados sólidos e semi-sólidos que resultam de atividades da comunidade de origem industrial, doméstica, hospitalar, comercial, agrícola, de serviços e de varrição. Ficam incluídos nesta definição os lodos provenientes de sistemas de tratamento de água, aqueles gerados em equipamentos e instalações de controle de poluição, bem como determinados líquidos cujas particularidades tornem viáveis seu lançamento na rede pública de esgotos ou 
corpos d’água ou exija para isso soluções técnicas e economicamente inviáveis, em face à melhor tecnologia disponível."

Schalch et al. (1990), classifica os resíduos de acordo com a sua procedência, ou seja, residencial, comercial, industrial, hospitalar, especial, de feira, varrição e outros.

Sendo assim, os resíduos de serviços de saúde - RSS, seriam diferenciados dos resíduos líquidos lançados nos esgotos domésticos e também da emissão de gazes na atmosfera feita através das chaminés.

A denominação resíduos de serviços de saúde foi adotada no Brasil pela ABNT (1993) a partir de dezembro de 1987 e firmada entre as definições da NBR 12807, com validade a partir de $1^{\circ}$ de abril de 1993 onde determina que resíduos de serviços de saúde correspondem àqueles resultantes de atividades exercidas por estabelecimento gerador, de acordo com a classificação adotada pela NBR 12808.

As questões relacionadas aos resíduos sólidos, conforme demonstramos, correspondem a um assunto muito amplo e complexo, pois englobam diferentes áreas de atuação, que vão desde o campo social até o financeiro, para que os mesmos possam ser conduzidos de forma adequada desde o local onde são gerados até o seu destino final, pois a presença do material orgânico, radioativo e perfuro cortante oferecem riscos à saúde e ao meio ambiente. (Couto, 1997)

Entre as definições sobre os resíduos de serviços de saúde - RSS, temos também, as definições apresentadas pela Organização Mundial de Saúde OMS, Associação Brasileira de Normas Técnicas - ABNT, Conselho Nacional do Meio Ambiente - CONAMA e Centro de Vigilância Sanitária - CVS.

A Organização Mundial de Saúde OMS define os resíduos de serviços de saúde como: "Resíduo de serviço de saúde, é todo aquele gerado por prestadores de assistência médica, odontológica, laboratorial, farmacêutica, instituições de ensino e pesquisa médica, relacionados à população humana bem como veterinário, possuindo potencial risco, em função da presença de materiais biológico capazes de causar infecção, produtos químicos perigosos objetos perfurocortante efetiva ou potencialmente contaminado e mesmo rejeitos radioativos, necessitando de cuidados específicos de acondicionamento, transporte, armazenamento, coleta e tratamento." (Andrade, 1995, p.3)

Entre todos os grupos de resíduos gerados, cada um tem o órgão responsável pelo seu gerenciamento adequado. $\mathrm{Na}$ tabela a seguir observamos os responsáveis pelo gerenciamento das diferentes classes de resíduos:
Tabela 01. Responsabilidade pelo gerenciamento de cada tipo de resíduo.

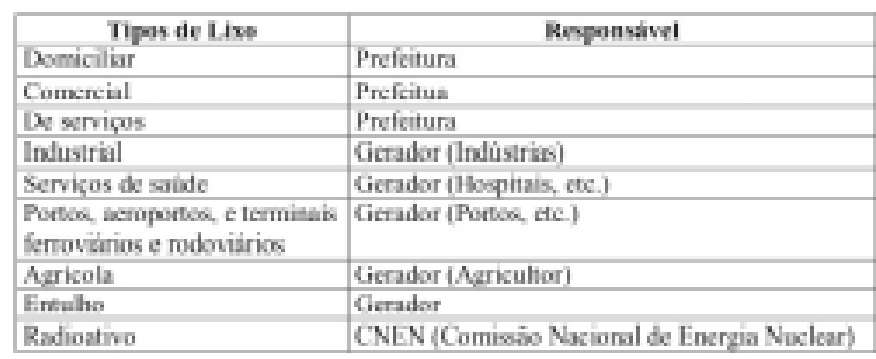

Fonte: Leite et al. (2002)

Conforme a resolução do CONAMA n ${ }^{\circ} 5$ de 5/8/93, em seu artigo $4^{\circ}$, fica determinado que é de responsabilidade dos estabelecimento prestadores de serviços de saúde "...o gerenciamento de seus resíduos sólidos, desde a geração até a disposição final, de forma a atender aos requisitos ambientais e de saúde pública." Conforme cita Schalch et al (1990), existe uma variação de uma unidade geradora para outra na composição dos RSS, que é influenciada por diversos fatores, entre eles as condições sociais do gerador, as condições climáticas, assim como os hábitos e costumes de cada gerador.

Os RSS correspondem a uma pequena parcela, se forem comparados aos demais resíduos sólidos, porém, consideram-se as suas principais características que correspondem a sua patogenicidade, colocando-os assim, dentro da classe I, ou seja, resíduos perigosos conforme determina a NBR 10.004

Alguns autores contestam a questão dos cuidados redobrados para os RSS, assim como vemos no trabalho de tese apresentado por Andrade (1997), onde cita que a maioria dos microorganismos encontrados nos RSS corresponde aos microorganismos pertencentes à microbiota normal humana, podendo também estar presente em outros resíduos como exemplo o resíduo domiciliar, sendo incapaz por si próprio de causar uma infecção, porém excluídos estão os perfurocortantes e outros infectantes. Mas sabemos que tais resíduos quer sejam eles de origem domiciliar ou hospitalar, não deixam de oferecer riscos ao meio ambiente.

A falta de informações sobre os RSS faz com que lhes sejam atribuídos, na maioria das vezes, pouca importância, ou ainda um excesso de cuidados desnecessários, gerando um aumento dos custos com os mesmos, assim como, muitas vezes as infecções hospitalares são atribuídas erroneamente à esses resíduos.

Segundo Fernandes (2000), podemos afirmar com segurança, que quando os RSS são gerenciados em condições ideais, o risco de transmissão de doenças aos profissionais da saúde diretamente ligados aos RSS são considerados muito baixos, enquanto que os riscos de transmissão de doenças aos pacientes e comunidade são praticamente nulo. 
Tabela 02. Classificação de resíduos de serviços de saúde em categorias segundo a NBR 12808/ABNT.

\begin{tabular}{|c|c|}
\hline Categoria & Constituintes \\
\hline \multicolumn{2}{|l|}{ CLASSE A - Residuos infectantes } \\
\hline Tipo A. I- Biológico & $\begin{array}{l}\text { cultura, inoculo, mistura de microorganismos e } \\
\text { meio de cultura inoculado proveniente de } \\
\text { labonatório clinico ou de pesquisa, vacina vencida } \\
\text { ou inatilizada, filtro de gases aspirados de áreas } \\
\text { contaminadas por agentes infectantes e qualquer } \\
\text { resíduo contaminado por estes materiais. }\end{array}$ \\
\hline $\begin{array}{l}\text { Tipo A.2 - Sangue e } \\
\text { hemoderivados }\end{array}$ & $\begin{array}{l}\text { bolsas de sangue após transfussio, com prazo de } \\
\text { validade vencido ou sorologia positiva, amostras de } \\
\text { sangue para análise, soro, plasma e outros } \\
\text { subprodutos. }\end{array}$ \\
\hline Tipo A.3 - Cirúrgico & $\begin{array}{l}\text { anatomopatobgico e exudato: tecido, órgào , féto, } \\
\text { peça anatômica, sangue e outros liquidos orgânicos } \\
\text { resultantes de cirurgia, necropsia e residuos } \\
\text { contaminados por estes materiais. }\end{array}$ \\
\hline Tipo A.4 - Perfurame ou cortante & agulha, ampola, pipeta, làmina de bisturi e vidro. \\
\hline Tipo A.5 - Animal contaminado & $\begin{array}{l}\text { carcaça ou parte de animal inoculado, exposto à } \\
\text { microorganismos patogénicos ou portador de } \\
\text { docnça infecto contagiosa, bem como residuos que } \\
\text { tenham estado em contato com este. }\end{array}$ \\
\hline Tipo A.6 - Assistćncia ao paciente & $\begin{array}{l}\text { Secrep̧ões, excreções e demais liquidos orgânicos } \\
\text { procedentes de pacientes, bem como os residuos } \\
\text { contaminados por estes materiais, inclusive restos } \\
\text { de refeiçồes. }\end{array}$ \\
\hline \multicolumn{2}{|l|}{ CLASSE B - Residuos especiais } \\
\hline Tipo B.1 - Rejeito radioativo & $\begin{array}{l}\text { material radioativo ou contaminado, com } \\
\text { radionuclideos provenientes de laboratórios de } \\
\text { análises clinicas, servicos de medicina e } \\
\text { radioterapia (ver Resoluçio CNEN-NE - } 6.05 \text { ). }\end{array}$ \\
\hline Tipo B.2 - Residuo farmacéutico & $\begin{array}{l}\text { medicamento vencido, contaminado, interditado ou } \\
\text { niio utilizado. }\end{array}$ \\
\hline $\begin{array}{l}\text { Tipo B.3 - Residuo quimico } \\
\text { perigoso }\end{array}$ & $\begin{array}{l}\text { residuo tóxico, corrosivo, inflamável, explosivo, } \\
\text { reativo, genotóxico ou mutagènico, conforme NBR } \\
10004 \text {. }\end{array}$ \\
\hline CLASSE C - Residuo comum & $\begin{array}{l}\text { todos aqueles que nâo se enquadram nos típos A e B } \\
\text { e que, por, sua semelhança aos residuos domésticos, } \\
\text { nåo oferecem risco adicional à saúde pública, por } \\
\text { exemplo, residuos de atividades administrativas, dos } \\
\text { serviços de varrição e limpeza de jardins e restos } \\
\text { alimentares que não entram em contato com } \\
\text { pacientes. }\end{array}$ \\
\hline
\end{tabular}

Fonte: ABNT - NBR 12.808/93.
Tabela 03. A classificação adotada pela resolução do CONAMA nº5/93, e a divisão dos RSS em quatro grandes grupos.

\begin{tabular}{|c|c|c|}
\hline & Categaria & $\begin{array}{c}\text { Canstituintes } \\
\end{array}$ \\
\hline Grupo A & $\begin{array}{l}\text { Resiluos que } \\
\text { apresentamin rbos } \\
\text { potencial à saíde } \\
\text { as meio aubiente } \\
\text { devido à presenca de } \\
\text { agentes biológioos }\end{array}$ & 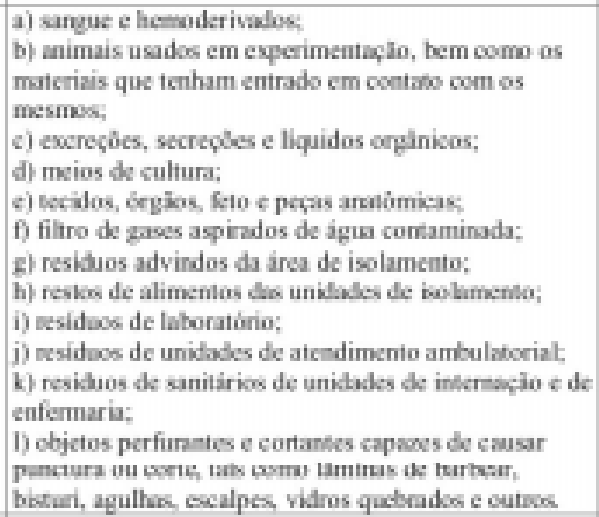 \\
\hline Girupo B & 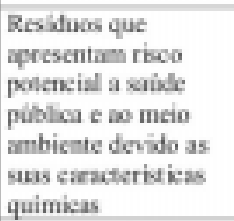 & 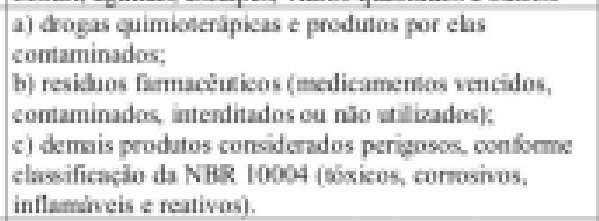 \\
\hline Grupo C & Rejeilos radioatinas & 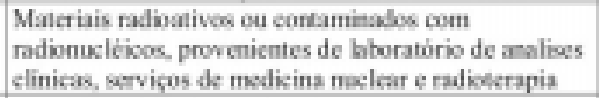 \\
\hline Grupo D & Residuos cremuns & 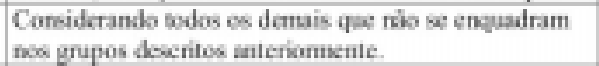 \\
\hline
\end{tabular}

Fonte: CONAMA, 1993.

Tabela 04. Classificação de resíduos de serviços de saúde pela categoria, segundo o Centro de Vigilância Sanitária - CVS - SUDS-SP.

\begin{tabular}{|l|}
\hline \multicolumn{1}{|c|}{ Categoria } \\
\hline A. Residuos infectantes \\
A.1. Material proveniente de locais de isolamento \\
A.2. Material biológico \\
A.3. Sangue humano e hemoderivados \\
A.4. Residuos cirúrgicos e anatomopatológico \\
A.5. Residuos perfurantes \\
A.6. Animais contaminados \\
B. Residuos especiais \\
B.1. Residuos radioativos \\
B.2. Residuos farmacẽuticos \\
B.3. Residuos quimicos perigosos \\
C. Residuos comuns \\
\hline
\end{tabular}

Fonte: CVS/SUDS, 1989 
Segundo Schneider (2001), existem alguns componentes dos RSS que merecem ser discutidos separadamente, pois suscitam dúvidas, em relação ao seu grau de infecção. Entre eles estão as peças anatômicas, frascos de medicamentos, fraldas descartáveis, absorventes higiênicos e papel higiênico e os resíduos alimentares.

As peças anatômicas são consideradas como infectantes, porém mantidas em solução de formol, para análise cito ou histopatológico ou anatomopatológico, ocorrendo assim à mudança de classe, pois a adição do formol à peça torna-a um resíduo químico perigoso em função das características químicas do produto adicionado, levando assim a dúvidas quanto a sua disposição final.

Os frascos de medicamentos, dentre eles os medicamentos vencidos, contaminados, interditados ou não utilizados são classificados como resíduos especiais, Classe I - Perigosos (NBR 10004/87), mesmo após serem utilizados os conteúdos das embalagens, conservam resíduos das soluções, o que conferem as mesmas a classificação de resíduos perigoso, principalmente por quimioterápicos, antibióticos ou outras drogas que ofereçam riscos ao meio ambiente. Os frascos de medicamentos, não são mencionados pela NBR 12.808/ 93 e nem pela Resolução CONAMA 05/93. A disposição final dos mesmos é discutida em todos os métodos disponíveis para tal, que sejam eles esterilização, aterro, ou incineração.

As fraldas descartáveis, absorventes higiênicos e papel higiênico estão incluídos na classe dos resíduos infectantes, pois se apresentam impregnados de fezes, sangue, secreções, excreções ou outros líquidos orgânicos patogênicos, já na classificação dos resíduos domiciliares os mesmos resíduos são denominados comuns. A preocupação se volta para o volume que estes resíduos representam, pois dentro da instituição de saúde estes resíduos também são gerados pelos profissionais atuantes, visitantes, familiares e demais pessoas que circulam no hospital

Os resíduos alimentares dos pacientes são considerados como infectante, porém os sistemas de controle de infecção hospitalar têm questionado em considerar infectantes somente os resíduos alimentares, nos casos dos pacientes em regime de isolamento por doenças infecto contagiosas e os demais resíduos dos pacientes fora deste regime, poderão ser gerenciados como resíduos comuns

Os resíduos oriundos de portos, aeroportos, terminais rodoviários e ferroviários, se enquadram na classificação dos resíduos sépticos, pois podem conter materiais patogênicos, tais como: materiais de higiene pessoal, restos alimentares assim como veicular doenças de outras cidades, estados ou mesmo país. (Leite, 2002)

Os resíduos de serviços de serviços de saúde são componentes representativos dos resíduos urbanos, não considerados pelo volume gerado, mais sim pelo risco que representam à população e ao meio ambiente. $\mathrm{O}$ manuseio e disposição final dos resíduos de serviços de saúde, se não forem bem gerenciados, representam um risco grave a saúde dos profissionais que estão direta e indiretamente ligados aos resíduos, assim como ao meio ambiente. (Scheneider, 2001)

\section{Histórico sobre os RSS}

A definição de resíduos de serviços de saúde, durante muitos anos foi questionada, recebendo diversos outros termos, como resíduo biológico, médico, clínico infectantes, e assim, aplicados ao longo do tempo, passando por diferentes formações de conceitos, chegando-se a conclusão que resíduos hospitalares e o temo sólido seriam designados aos perfuro cortantes, e também somente seriam considerados os resíduos oriundos de ambientes hospitalares.

Historicamente, vemos que no ano de 1920, ainda não havia publicações sobre os resíduos de serviços de saúde, vindo à tona somente em 1930, época esta em que se questionava sobre o que fazer com os resíduos hospitalares. Já na década de 40, foi adotado o incinerador como forma única de eliminar os resíduos hospitalares, mesmo com suas inúmeras desvantagens. (Scheneider, 2001)

Nos anos 50, voltam-se as atenções para o os resíduos radioativos, assim com surgem às preocupações de como manejá-los adequadamente.

$\mathrm{Na}$ década de 60 , as preocupações ampliam-se para o controle mais rigoroso que venha a impedir a dispersão das doenças infecto contagiosa, assim como a dispersão de gases na atmosfera através dos resíduos lançados durante o processo de incineração. Nesta década os resíduos sólidos passaram a ser de interesse público e governamental.

Em 1969, com o ato nacional sobre o meio ambiente, os resíduos passaram a fazer parte das metas do governo Federal, que assumiu o compromisso de manter a qualidade do meio ambiente, consolidado pelo Conselho de Qualidade Ambiental. (Schalch, 2001)

O aumento do uso dos descartáveis, na década de 70 , desperta para as preocupações de como armazenar tais resíduos, e o uso mais efetivo dos Equipamentos de Proteção Individual - EPI, luvas e máscaras, ganham destaque, com o aparecimento de novas patologias, entre elas a Síndrome da Imuno Deficiência Adquirida (SIDA). (Scheneider, 2001)

As questões sobre o gerenciamento dos RSS, nos EUA, são originadas de órgãos governamentais independentes, apresentando divergências em sua compreensão, sendo utilizado três definições, sem levar em conta as diversas definições locais e estaduais. (Schalch, 2001)

EPA - Eviromental Protection Agency, criada em 2 de dezembro de 1970, sendo a mais ativa entre todas até os dias atuais, ou seja a Agencia Federal com maior autoridade para administrar os resíduos infecciosos e perigosos, com representação em diversos estados americanos, e com reconhecimento internacional. A EPA defini os resíduos de serviços de saúde 
como qualquer resíduo sólido gerado do diagnóstico, tratamento ou imunização de pessoas, animais, e de material de pesquisa, assim como testes de agentes biológico, não incluindo, portanto, nenhum tipo de resíduo perigoso ou domiciliar.

CDC - Center for Disease Control, ligado ao Department of Health and Human Services - HHS, que em 1987, editou os procedimentos para controle de infecções, tratados hoje como precauções universais, protegendo assim o trabalhador da saúde, classificando os resíduos infecciosos em quatro categorias resíduos de laboratório e microbiologia, sangue e hemoderivados, resíduos patológicos e resíduos cortantes

OSHA - Ocupacional Safety and Health Administration, é a agência do DOL - Department of Labor, atuante na saúde do trabalhador considerando o contato ocupacional como o maior meio em potencial para infecção nos mesmos, sendo um dos seus papéis, manter o trabalhador informado sobre os riscos aos quais está exposto.

\section{Gerenciamento dos RSS}

O processo de gerenciamento dos RSS é abrangente, envolve várias etapas desde a geração, segregação, acondicionamento, transporte e destino final.

A segregação dos RSS, quando bem administrada, oferece benefícios à fonte geradora, como a economia dos coletores a ele destinado. Considerandose que a segregação dos resíduos infectantes poderiam chegar a índices de $15 \%$ em nosso país nas unidades bem estruturadas, porém, o que se observa é que este valor não ultrapassa aos $30 \%$, lembrando que o aumento ou a diminuição destes valores depende do interesse e organização dos profissionais da instituição que estão envolvidos no processo. (Fernandes, 2000)

É importante a conscientização das pessoas quanto ao uso da terminologia correta para os RSS, inclusive das classes e tipos de resíduos, já que o mesmo é composto pelos resíduos infectantes, especiais e comuns.

A segregação dos RSS deve ser realizada no ambiente onde são produzidos, não sendo permitido o seu manuseio quando já dispostos seus conteúdos, em embalagens, evitando assim possíveis acidentes.

O acondicionamento dos RSS é determinado pela NBR 9.190, que determina as características importantes como dimensões, resistência, cor e identificação, sendo estas características monitoradas pelas análises realizadas por laboratório especializado

Os resíduos comuns são acondicionados em embalagens plásticas de qualquer cor, exceto a cor branca que fica reservada para os resíduos infectantes, que ainda devem conter o símbolo internacional conforme NBR 7.500, assim como os dados de identificação ao fabricante junto à solda inferior da embalagem plástica. (Fernandes, 2000)

Os resíduos perfurocortantes devem ser acondicionados em embalagens rígidas, conforme determina a ABNT. Sabemos, portanto, que são utilizados na maioria das instituições, coletores de papelão que são frágeis quando expostos a umidade, sendo, porém recomendado coletor de material rígido, resistente a umidade e às possíveis perfurações por agulhas, e com diâmetro de abertura suficiente para a passagem dos resíduos, sem oferecer riscos aos profissionais que o manuseiam.

O manuseio dos resíduos infectantes implica contato direto em todas as etapas, que compreendem desde a coleta interna até o transporte e destino final, já que todo o processo é realizado manualmente.

$\mathrm{O}$ descumprimento ou desconhecimento do manuseio adequado dos resíduos infectantes levam o profissional que tem contato com os RSS a uma exposição maior aos riscos ocupacionais, considerando que os funcionários da coleta são aqueles mais expostos aos agentes presentes nos resíduos, pois o manuseiam desde o local onde são gerados até o seu acondicionamento final dentro da instituição, sendo os profissionais da saúde, principalmente a equipe de enfermagem o segundo grupo mais exposto aos riscos mencionados.

A atenção que a Comissão de Controle de Infecção Hospitalar - CCIH dentro da instituição hospitalar destina às questões de qualidade e biossegurança, devem ser direcionadas não somente aos pacientes, mas também aos demais profissionais da saúde ligados diretamente ao paciente, ampliando-se para os prestadores de serviços, pessoas que circulam interna ou externamente no hospital, para que não fiquem expostas às contaminações por contato com os resíduos infectantes. (Fernandes, 2000)

Outro fator de risco que devemos considerar é o mau uso dos Equipamentos de Proteção Individual - EPI, assim como o acondicionamento inadequado dos mesmos, e o não cumprimento das medidas básicas de higiene.

Para evitar tais acidentes, todas as atividades relacionadas ao manuseio dos RSS, devem ser padronizadas e detalhadas por escrito passo a passo com linguagem clara e acessível a todos os profissionais, em manual de normas e rotinas para cada um dos procedimentos realizados, lembrando que para a elaboração deste manual, devem participar representantes de cada uma das classes envolvidas, entre eles a CCIH e representante administrativo.

Quando se trata das questões de biossegurança, os equipamentos de proteção individual - EPI, são indispensáveis, pois correspondem à única barreira de segurança do profissional contra a contaminação através dos RSS.

Entre os EPI existentes, destacamos aqueles mais usados no ambiente hospitalar, máscara, luva, óculos de proteção, avental de manga longa, gorro, pró pé e botas de borracha de cano longo, devendo ser sempre retirados após o uso indicado conforme a situação, destinando os descartáveis ao local adequado para o seu acondicionamento e os de uso permanente deverão ser encaminhados ao setor específico para a devida limpeza e posterior acondicionamento É de responsabilidade do gerador o fornecimento dos EPI necessários aos 
profissionais, assim como o treinamento e supervisão de uso dos mesmos

As áreas hospitalares são classificadas segundo a Portaria 930/92 do Ministério da Saúde, onde especifica o risco que cada uma oferece através dos resíduos que são gerados: áreas críticas, áreas semi-críticas e áreas não críticas.

As áreas críticas são aquelas onde o risco de transmissão de infecção é maior, pois corresponde a áreas onde o volume de procedimentos de risco é maior, como exemplo as salas de cirurgia, UTI, pronto socorro, unidade de queimados, isolamentos e outros.

As áreas semi-críticas são áreas ocupadas por pacientes com patologias de baixa transmissibilidade e doenças não infecciosas,como exemplo enfermarias, quarto de pacientes internados e ambulatórios.

As áreas não críticas correspondem as não ocupadas por pacientes, como exemplo as áreas administrativas. (Fernandes, 2000; Andrade, 1997)

\section{Os RSS e a questão ambiental}

Verificamos que, dentre os diferentes tipos de resíduos gerados pelo homem, os produzidos pelos serviços de saúde - RSS, merecem particular atenção, devido, principalmente, ao elevado potencial de risco de contaminação, que oferecem não somente ao ambiente onde os mesmos são gerados mas também do meio externo onde são dispostos no meio ambiente

Vemos na tabela abaixo, exemplo de alguns vetores e as doenças transmitidas por eles.

Tabela 05. Doenças transmitidas por vetores encontrados nos RSS.

\begin{tabular}{|c|c|}
\hline Mosca & $\begin{array}{l}\text { Febre tifoide } \\
\text { Salmoselose } \\
\text { Disemeria }\end{array}$ \\
\hline Mosca & $\begin{array}{l}\text { Febre tif̂ide } \\
\text { Salmonselose } \\
\text { Disemeria }\end{array}$ \\
\hline Mosquite & $\begin{array}{l}\text { Maliria } \\
\text { Febre amarela } \\
\text { Dergue }\end{array}$ \\
\hline Barata & $\begin{array}{l}\text { Febre tifoide } \\
\text { Cólera } \\
\text { Amebiase } \\
\text { Giaedlase }\end{array}$ \\
\hline Rato & $\begin{array}{l}\text { Tiso Murino } \\
\text { Leptospirose } \\
\text { S2 Imoselose } \\
\text { Triquinose } \\
\text { Peste bulonica } \\
\text { Febre da mordda do fato } \\
\text { Diaretelas e desenterias }\end{array}$ \\
\hline Poneo & Cisticencose \\
\hline
\end{tabular}

Fonte: Fernandes, 2000, p.1160.
Os microvetores também presentes nos resíduos, quando em condições favoráveis, têm a sobrevida mantida, em períodos que variam de dias a anos conforme vemos descritos na tabela abaixo.

Tabela 06. Tempo de sobrevivência de microvetores encontrados nos RSS

\begin{tabular}{|l|c|}
\hline \multicolumn{1}{|c|}{ Organismo } & Tempo (dias) \\
\hline Enfoameba histolytica & $8-12$ \\
\hline Leptospira interrogans & $15-43$ \\
\hline Polivirus & $20-170$ \\
\hline Larvas de vermes & $25-40$ \\
\hline Salmonella typhi & $29-70$ \\
\hline Bacilo da tuberculose & $150-180$ \\
\hline Áscaris lumbricoides & $2.000-2.500$ \\
\hline
\end{tabular}

Fonte: Torres, 2001.

As diversas pesquisas onde são realizadas as análises microbiológicas nos RSS, tendem a justificar os cuidados com estes resíduos. Os resultados demonstram que a carga bacteriana dos RSS são menores, inclusive dos infectantes, considerando que no descarte os mesmos se misturam a outras soluções já aderidas como, por exemplo, as ataduras de curativos, promovendo assim a diluição da carga de microorganismos existentes reduzido-os ou até mesmo anulando-os. (Fernandes, 2000)

Verificamos, portanto, que a discussão teórica sobre os RSS nos proporcionou um embasamento necessário para a pesquisa de campo que já foi realizada com os profissionais da área da saúde - enfermeiros, técnicos de enfermagem e auxiliares de enfermagem do Hospital Beneficência Portuguesa de Araraquara, visando um estudo de percepção do ambiente de trabalho desses profissionais que estão intimamente ligados aos RSS e à percepção do meio ambiente. Os resultados dessa pesquisa serão tema de artigo futuro.

\section{Referências bibliográficas:}

ANDRADE, J.B.L. Análise do fluxo e das características físicas, químicas e microbiológicas dos resíduos de serviços de saúde: proposta de metodologia para o gerenciamento em unidades hospitalares. São Carlos. 208p. Tese (Doutorado) - Escola de Engenharia de São Carlos, Universidade de São Paulo, 1997. 
BRASIL - ABNT. Associação de Normas Técnicas - NBR 12808:

Resíduos de Serviços de Saúde: Classificação. São Paulo, 1993.

BRASIL - Resolução CONAMA n ${ }^{\circ} 5$ de 5 de agosto de 1993. Define as normas mínimas para tratamento de resíduos sólidos oriundos de serviços de saúde, portos, aeroportos e terminais rodoviários e ferroviários. Diário

Oficial da República Federativa do Brasil. Brasília (DF), no 166, 31 de agosto 1993, seção I, p.12997.

COUTO, R. et al. Infecção hospitalar epidemiologia e controle. Rio de Janeiro: Medsi, 1997.

FERREIRA, A.B.H. Novo Aurélio Século XXI: o dicionário da língua portuguesa. 4. imp. Rio de Janeiro: Nova Fronteira, 1999.

FERNANDES, A. T. Infecção hospitalar e suas interfaces na área da saúde. São Paulo: Atheneu: 2000, p.1721.

LEITE, W.C.A. et al. Gestão e tecnologias de tratamento e disposição de resíduos sólidos. São Carlos. Escola de Engenharia de São Carlos,

Universidade de São Paulo, 2002.

SUDS - São Paulo. Subsídios para organização de sistema de resíduos em serviços de saúde. 1989.

SCHALCH, et al. Resíduos de serviços saúde. In: Curso sobre

Gerenciamento de Resíduos Sólidos. Goiânia, Associação Brasileira de Engenharia Sanitária e Ambiental, 1990, p.209.

SCHNNEIDER, V.E. et al. Manual de gerenciamento de resíduos sólidos de serviços de saúde. São Paulo: CLR Balieiro. 2001, p.173

SISINNO, C.L.S.; OLIVEIRA, R.M. (Orgs). Resíduos sólidos, ambiente e saúde: uma visão multidisciplinar. Rio de Janeiro: Editora Fiocruz, 2000, p.137.

TORRES, S. et al. Limpeza e higiene. Lavanderia hospitalar. 2.ed. São Paulo: Balieiro, 2001.

\section{Resumo:}

Nas últimas décadas, a discussão da geração dos resíduos relacionados às questões ambientais têm sido objeto de estudo de muitas pesquisas. Dentre os resíduos, os resíduos de serviços de saúde - RSS, são vistos como problemas que envolvem, além da área ambiental, outras áreas da sociedade como a saúde pública, o saneamento básico, a qualidade de vida da população quando gerenciados de forma inadequada. Parte dessa discussão origina-se no âmbito dos estabelecimentos destinados aos cuidados da saúde, tais como hospitais, laboratórios, consultórios médico-odontológicos, dentre outros. Destacam-se aqueles onde o número de atendimentos e procedimentos é maior, conseqüentemente, gerando um maior número de resíduos, principalmente nos hospitais. Os profissionais da saúde que realizam todos os procedimentos nos pacientes e estão diretamente em contato com os RSS devem ter consciência dos prejuízos que o mau gerenciamento poderá acarretar. A discussão teórica sobre os RSS visa um entendimento para necessária pesquisa de campo, com objetivo de conscientização do profissional da área de saúde voltada à relação dos RSS e a questão ambiental, que atenda as expectativas de se manter uma qualidade de vida ideal para o profissional, população e a preservação do meio ambiente.

\section{Palavras-chave:}

Resíduos de Serviços de Saúde, Meio Ambiente, Gerenciamento. 